\title{
Une laiterie administrative à Tahiti
}

\author{
par P. BESNAULT
}

\section{ÉLABORATION DU PROJET}

La pénurie de lait frais et de produits laitiers à Tahiti et spécialement à Papeete, qui compte avec sa zone suburbaine plus de 15.000 habitants, a depuis longtemps attiré l'attention de l'Administration et de certains particuliers. Pendant la guer re un éleveur américain installa, dans de bonnes conditions d'hygiène, une laiterie destinée surtout à alimenter l'hôpital et la maternité. La récolte du lait s'y fait mécaniquement, le lait est refroidi, tout au moins celui de la traite du soir qui est conservé jusqu'au lendemain matin. Depuis, deux éleveurs français ont suivi cet exemple, comprenant l'intérêt qu'il y a à approvisionner les particuliers de la ville. Progressivement ils ont commencé à traire, à accroître et à améliorer leurs troupeaux. Ces trois élevages situés à 3,4 et 10 kilomètres de Papeete livrent environ 300 litres de lait par jour.

Cependant le manque de lait était toujours sensible aux périodes de sécheresse qui correspondent généralement à la fin de la lactation. Les vêlages sont nombreux aux environs de Noël et la sécheresse se manifeste sur l'état des pâturages à partir du mois d'août.

C'est pourquoi malgré de nombreuses oppositions et grâce à l'intérêt qu'y porta Monsieur le Gouverneur Anzianni, une laiterie administrative fut inscrite au programme d'équipement du Service de l'Élevage au titre du FIDES.

Le projet comportait deux éléments essentiels : le troupeau et l'installation de traitement du lait. Il fut décidé que les vaches laitières seraient confiées à la station d'altitude du Service de l'Élevage dans la presqu'île de Taravao. Le Service dispose là d'un domaine de 200 hectares, encore en grande partie à défricher, en zone beaucoup plus humide que la région de Pirae où sont la station mère et le siège du Service.

La distance de 60 kilomètres entre le lieu de production et le lieu de consommation est un inconvénient certain, mais permet d'espérer la réalisation d'un service de collecte du lait des élevages échelonnés sur le parcours. Le centre de traitement et de conditionnement du lait est, lui, à Pirae, dans la zone de consommation. La plus grande partie du lait livré en ville est centralisée et mise en bouteilles à Pirae. Notre ambition est naturellement de pasteuriser tout le lait vendu en ville. Le centre de pasteurisation actuellement existant serait tout à fait suffisant.

\section{LE TROUPEAU}

La première souche du troupeau laitier du Service était constituée par 6 vaches Friesian-Holstein et 5 Jersey importées de Nouvelle-Zélande en 1947. Ces animaux étaient jusqu'ici principalement exploités pour la production de jeunes géniteurs destinés à l'élevage privé. Le lait produit en, excès était consommé sur place. Les résultats avaient été bien meilleurs avec les Holstein qu'avec les Jersey et il fut décidé de ne pas persévérer avec cette race. Malgré la bonne origine des Jersey importées, leur production fut toujours médiocre (4 à 5 litres de lait par jour); la mortalité des jeunes fut toujours élevée et la carcasse à la réforme manque de poids. Le choix se porta donc, pour l'important achat prévu en Nouvelle-Zélande, sur la race Holstein dont le rendement avait souvent dépassé 20 litres de lait avec le premier lot.

La maison Donald, d'Auckland, qui a une succursale à Papeete, fut chargée de l'importation de 30 génisses pleines. Les caractéristiques imposées étaient les suivantes : génisses primipares, inscrites au HerdBook, dont la mère devait avoir fourni au moins 5.000 litres de lait en une lactation, présentant une réaction négative à la tuberculine et une séroagglutination négative à l'antigène brucellique.

Les animaux, transportés par le navire Waitemata, furent débarqués le 28 décembre 1950. Il n'y avait pas eu le moindre incident pendant le voyage; ils étaient d'ailleurs aussi bien installés que possible : sur le pont, en box individuels.

A leur arrivée les génisses sont immédiatement conduites à la station de Taravao où elles sont mises en liberté dans un petit parc muni d'un abri. Elles continuent à recevoir, en plus du fourrage vert de prairie qu'elles pâturent, le foin sec et les aliments concentres qu'elles mangeaient à bord.

Elles sont parquées en zone fortement infestée de piroplasmose ( $P$. bigeminum) et ne seroni pas 


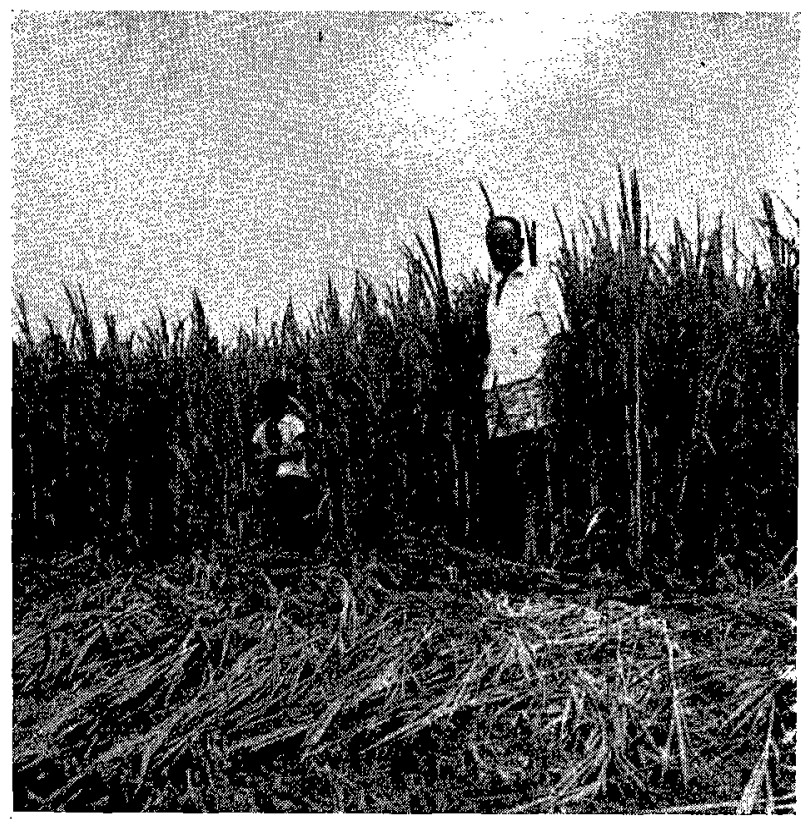

Champ d'elephant grass

Cette plante fourragère fournit la base de la ration donnée à l'étable. Les rendements sont très élevés

mises au bain arsenical avant d'être prémunies.

A partir du cinquième jour après leur importation, la température des vaches est prise matin et soir. Elles sont isolées quand la température atteint $40^{\circ}$ et traitées à la gonacrine $\left(1,2 \mathrm{~g}\right.$ dans $20 \mathrm{~cm}^{3}$ d'eau distillćc on injection intraveineuse) ou au zothélone $\left(6 \mathrm{~cm}^{3}\right)$ lorsque la température atteint $41^{\circ}$ ou que des signes cliniques se manifestent. La première génisse est cliniquement infestée le septième jour après le débarquement et en trois semaines, 23 animaux sur 30 ont eté infestés naturellement. Une génisse est morte après douze heures de maladie malgré une intervention précoce à la gonacrine.

Les 7 animaux encore indemnes sont inoculés avec du sang d'infestés chroniques. Un taurillon guéri depuis un mois d'une crise de piroplasmose et 2 vaches dont la maladie était plus ancienne, tous trois ayant été infestés naturellement et non traités, sont donneurs de virus; leur sang citraté, mélangé en parties égales, est injecté immédiatement sous la peau à raison de $5 \mathrm{~cm}^{3}$ par génisse. Les animaux inoculés font une crise fébrile le cinquième ou sixième jour généralement moins grave que la maladie naturelle et ne nécessitant pas toujours de thérapeutique.

Un mois après le débarquement on peut considérer tout le troupeau comme prémuni avec une 'seule perte (1). La surveillance peut être moins étroite et le troupeau est mis au pâturage.

Les génisses avaient été saillies en Nouvelle-Zélande; les deux tiers sont pleines, un tiers demandera le taureau à nouveau. Le premier vêlage a lieu en mai et le $l^{\text {er }}$ septembre, la laiterie commence à fonctionner.

\section{LA LAITERIE}

Le batiment de traite est un simple hangar dont 'le sol est cimenté, équipé de stalles métalliques de la Société nouvelle des Établissements Sarron; dites "cornalyses La Chicago » qui se sont révélées d'un usage parfaitement pratique.

Un local pour le réfrigérateur, la machine à traire et le stockage des pots vides, un parc à veaux, une fosse à purin sonl annexés au bâtiment de traite proprement dit.

Pour la préparation des aliments; un

(1) Les importations de bétail sont considérées comme difficles à Tahiti. Un éleveur, pendant la yuerre, perdit 13 vaches importées sur 18 à la première crise de piroplasmose. Nous sommes aujourd'hui mieux armés.

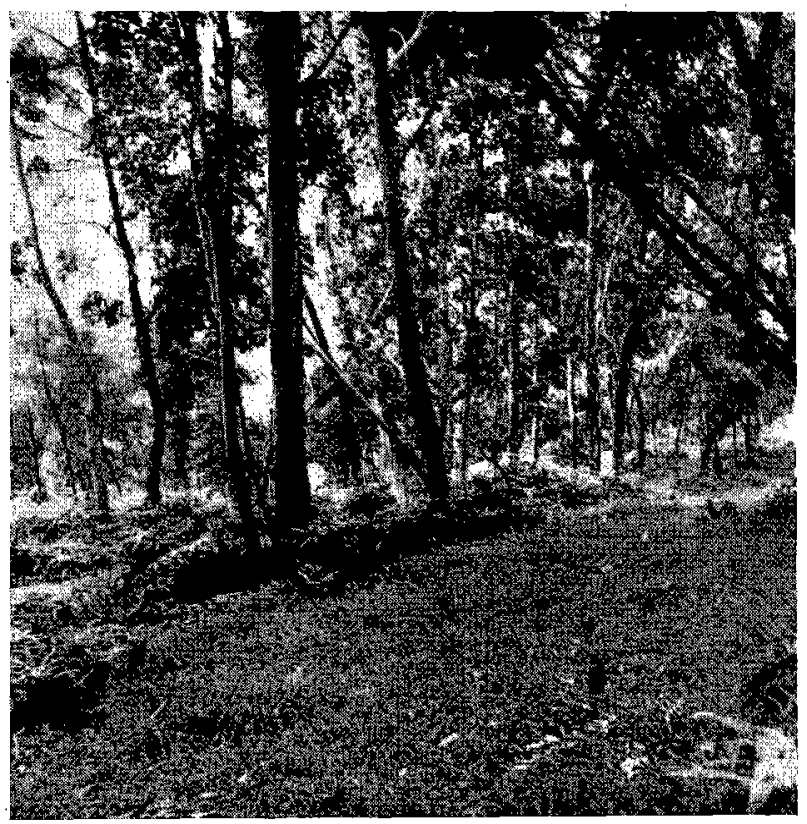

Pâturage artificiel de Melinis minutiflora sous eucalyptus. Les arbres sont plantés depuis une dizaine d'anrées. Ces plateaux stériles étaient couverts de fougères (Gleichenia linearis), l'humus des arbres a permis les semis de malinis qui remplace presque partout la fougère. 
broyeur à marteaux Gondard et une ensileuse Mac Cormick sont utilisés.

La traite est faite mécaniquement avec la machine portative « Universal » extrêmement simple qui recueille le lait directement dans les pots utilisés pour le transport. Le lait ne subit aucune manipulation à l'air libre jusqu'au pasteurisateur. L'entretien en est très simple puisqu'il ne comporte que le lavage des tétines de traite et des deux tubes collecteurs de 1,5 mètre qui peuvent être parfaitement brossés intérieurement. Deux fois par jour la machine

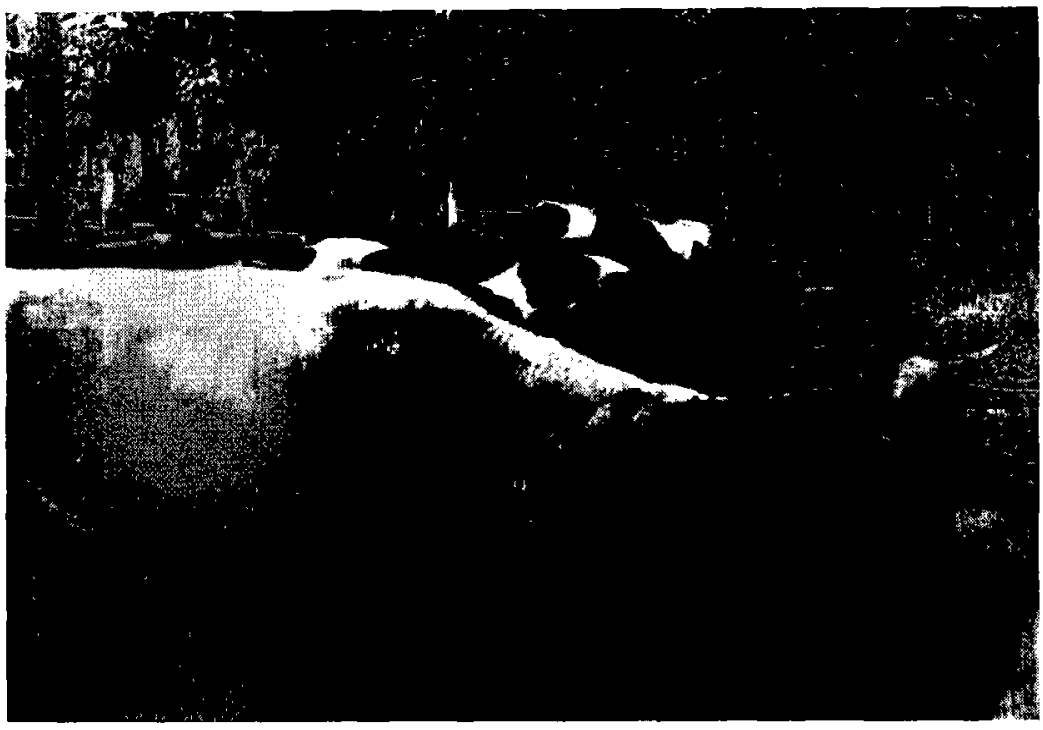
est nettoyée à la soude caustique qui donne de meilleurs résultats que le carbonate de soude (Voir le bulletin no 18 du Department of Agriculture, de Nouvelle-Zélande : « Claening of milking machines», par G.-M. Moir). Après

brossage, la machine est abondamment rincée à l'eau froide, elle aspire ensuite un seau d'eau bouillante contenant une cuillerée à café rase de soude caustique, puis un autre seau d'eau bouillante pure (pour deux jeux de tétines).

Entre les traites, les eléments de caout-

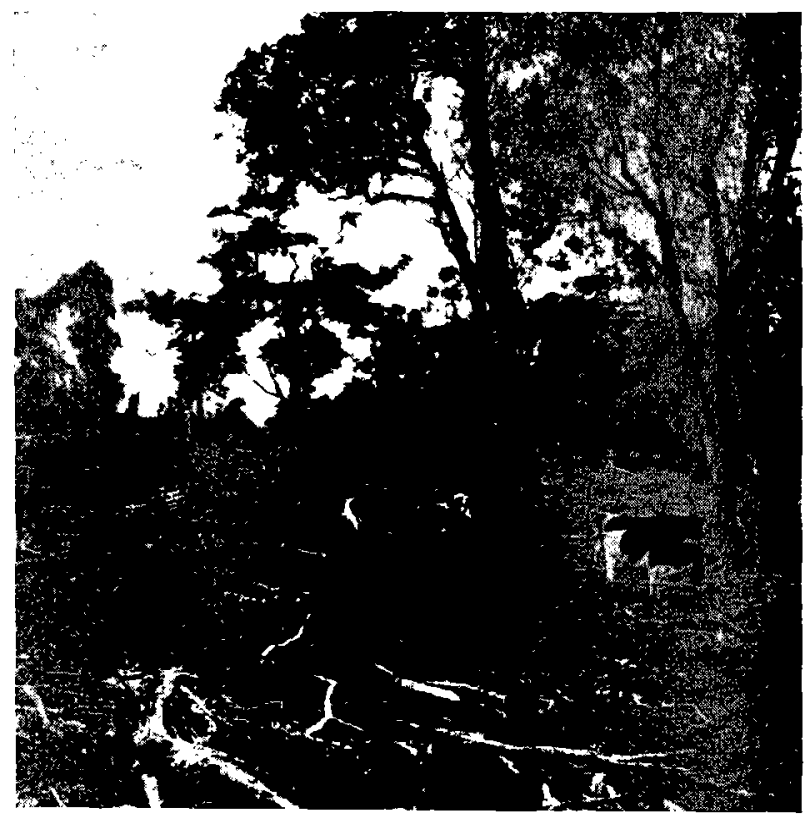

Paturage naturel

La forêt primitive a été abatuue (Mahame, Glochicion Tuhitense BaillouMara, Nauclea Forsteriana, Seem. - Purau, Hibiscus tiliaceus, $L$ in. - Fougères arborescentes, Cyato affinis Forster). Seuls quelgues arbres d'ombrage ont été conservés. Les mauvaises herbes sont débroussées de temps à autre. Le Paspalum conjugatum et le Commelina nodiflora tous deux fourragers, dominent. chouc sont suspendus remplis d'une solution javellisée faible (une cuillerée à soupe pour 20 litres d'eau).

Le lait est refroidi dans les pots dans un réfrigérateur américain de marque « International ». Les pots sont plongés dans l'eau glacée du réservoir constamment agitée. Le modèle utilisé est d'une capacité de 12 pots de 20 litres. Le lait fraichement trait est porté à la température de $4^{\circ}$ en quarante minutes. Il est transporté à la station de pasteurisation en caisses isothermes chargées au départ d'un bloc de glace de 20 kilos (caisses de 6 pots).

A chaque traite les vaches laitières reçoivent une ration composée de 10 kilos de fourrage vert haché (herbe à éléphant) et de 1 kilo de tourteau de coprah. Quatre hectares d'herbe à éléphant en zone très humide, fumés par le purin de la laiterie fournissent abondamment la ration de 30 à 40 laitières en 4 à 5 coupes annuelles.

\section{LA PASTEURISATION}

Lc lait est transporté à $4^{\circ}$, par camion Renault 2 tonnes, à la station de pasteurisation voisine de la ville. 


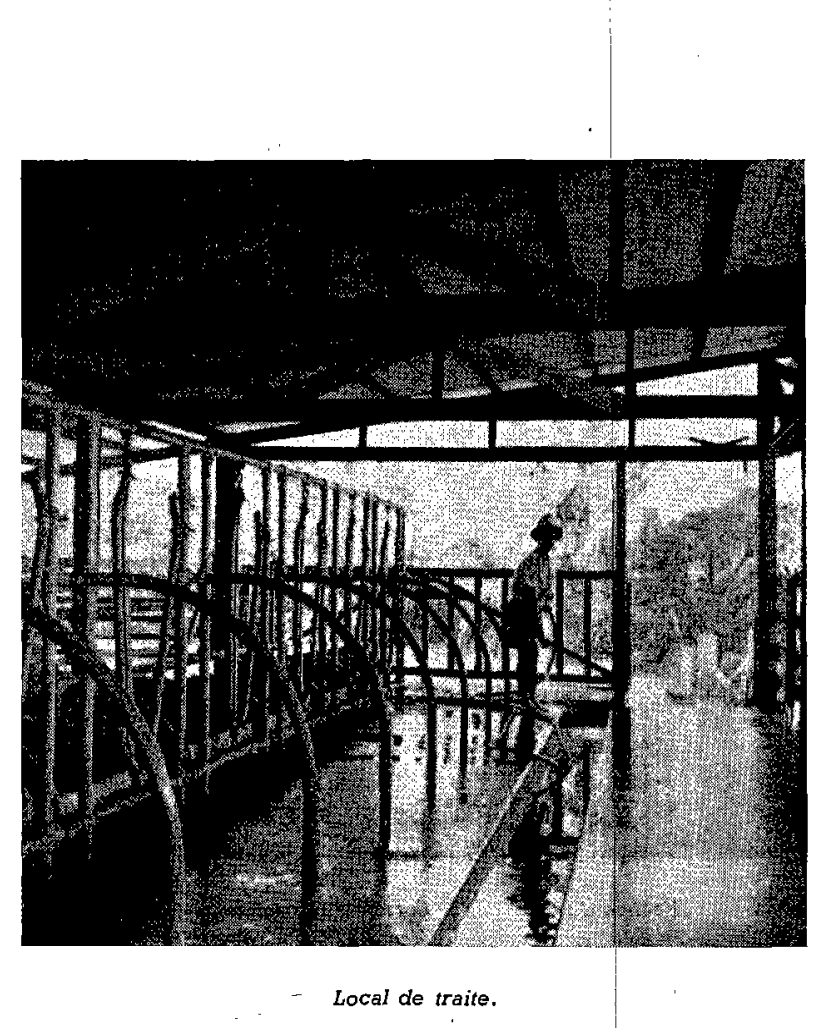

La vapeur utilisée pour la pasteurisation la stérilisation des pots; le lavage des bouteilles, etc., est fourni par une chaudière automatique à mazout "Kisko ».

\section{RÉSULTATS}

Les rendements en lait de primipares ayant supporté pendant la gestation un voyage en mer de trois semaines, une infestation de piroplasmose et une crise d'acclimatement sévère (elles sont arrivées à la période la plus chaude de l'année) ne sont pas très élevés; la moyenne de production journalière ne dépasse pas 10 litres. Mais les animaux ne peuvent être jugés à leur première lactation.

Cependant, depuis le ler $^{\text {er }}$ eptembre 1951, un apport de lait supplémentaire journalier de 200 litres a pu être fourni à la ville. Il est paradoxal de constater que la clientèle ne s'est pas immédiatement intéressée à la possibilité d'obtenir un produit dans des conditions d'hygiene nettement améliorées; une augmentation de prix d'un franc C.F.P. par litre

L'opération de pasteurisation demande environ une heure. L'appareil utilisé est de marque américaine (Dairy Package). Le chauffage et le refroidissement sont discontinus. Le réservoir contient environ 500 litres de lait qui sont portés très rapidement à la température de $64^{\circ}$ par circulation de vapeur d'eau sous pression dans la double paroi de la cuve. Cette température est maintenue pendant trente minutes et abaissée à $7^{\circ}$ ou $8^{\circ}$ en vingt minutes par circulation d'eau glacée.

L'opération est contrôlée par un thermomètre enregistreur.'

Le lait est conditionné en bouteilles standard stérilisées à l'eau javellisée après brossage et savonnage et livrées immédiatement au domicile du consommateur.

Les pots sont brossés au savon et stérilisés à la vapeur d'eau sous pression avant d'être réexpédiés à la laiterie. a suffi à la rendre réticente. Seuls quelques européens, progressivement, sont devenus clients de notre laiterie. Le supplément a été livré gratuitement au Service Social (20 à 40 litres par jour); ce qui était parfaitement dans l'esprit du programme de l'Administration au moment où le projet de laiterie avait été mis sur pied.

Ce ne sont que des considérations financières qui nous obligèrent à commercialiser le lait.

Les résultats de la distribution d'un verre de lait chaque matin aux enfants les plus déficients a été très favorable. Un rapport du Service Social en témoigne.

Une dernière étape reste à franchir; c'est l'obligation de pasteuriser tout le lait fourni à la ville. Elle sera ordonnée dès que la machine emplisseuse capsuleuse et les 2.000 carafes actuellement en commande seront débarquées. Le matériel existant pourra y suffire sans grands frais supplémentaires. 